\title{
Benign Versus Malignant Vertebral Compression, Chemical Shift MR Imaging, Is It Useful?
}

\author{
Gulgun Yilmaz Ovali, ${ }^{1,}$, Fatih Düzgün, ${ }^{1}$ Mustafa Farasat,, and Sebnem Orguc ${ }^{1}$ \\ ${ }^{1}$ Department of Radiology, Medicine School, Celal Bayar University, Manisa, Turkey \\ ${ }^{2}$ Manisa Merkez Efendi Goverment Hospital Radiology Department, Manisa, Turkey \\ "Corresponding author: Gulgun Yilmaz Ovali, Radiology Department, Medicine School, Celal Bayar University, Manisa, Turkey. Tel: +90-5327989618, Fax: +90-2362349070, \\ E-mail: gulgunyilmaz@hotmail.com
}

Received 2016 September 02; Revised 2016 October 29; Accepted 2016 November 14.

\begin{abstract}
Objectives: The aim of this study was to evaluate the diagnostic accuracy of chemical shift imaging in vertebral compression. Patients and Methods: Forty-nine patients with vertebral compression with suspected malignancy or history of trauma were included in this study. MR imaging of the spine with standard conventional MR sequences and additional chemical shift imaging was done with 1.5 Tesla MR Unit. Regions of interest (ROI) were placed on the abnormal marrow of compressed vertebrae both on the opposed phase and on the matching in phase images and signal intensity (SI) ratio values (SI out-phase/SI-in phase) were calculated. Results: Forty-nine patients had 68 spine lesions, consisting of 49 benign and 19 malignant fractures. Student $t$ test, receiver operating characteristic (ROC) analysis and interclass correlation test were used statistically. Mean SI ratio of benign vertebral compression ( $0.68 \pm 0.29$, range $0.13-1.53$ ) was significantly lower than malignant SI ratio values (1.06 \pm 0.10 , range $0.96-1.35)$. With student t-test, there was significant difference between benign compressions compared to malignant compressions $(\mathrm{P}<0.001)$. Receiver operating characteristic (ROC) curve analysis was used to determine the optimal cut off value in malignant and benign lesion definition. The optimal SI ratio cut off value was found to be 0.96 for separating benign and malignant vertebral compression. The area under the curve (AUC) value was observed as 0.92 (95\% confidence interval $[\mathrm{CI}]=0.86-0.99)$. According to that cutoff value, sensitivity was $100 \%(95 \% \mathrm{CI}=82.35 \%-100 \%$ ) and the specificity was $86 \%$ (95\% CI $=72.76 \%-94.06 \%$ ). Interobserver reliability was studied with intraclass correlation and results were statistically significant with intraclass correlation coefficient (ICC) as 0.85 (P $<0.05$ ). Conclusions: There is significant difference in signal values between benign and malignant compression fractures in chemical shift MR imaging. Chemical shift MR imaging has much additive data to conventional MRI in vertebral compression.
\end{abstract}

Keywords: Vertebral Compression, Chemical Shift Imaging, Spine, MRI

\section{Background}

Both malignant and benign vertebral compressions may show abnormal signal intensity changes in conventional MR images that may be similar and cannot be differentiated from each other. Normal bone marrow consisting of mainly fat should be found in benign compression; whereas, bone marrow infiltration in malignant ones leads to loss of fat. Defining fat in terms of signal loss in chemical shift imaging may differentiate these two entities, which have very different treatment algorithms (1).

\section{Objectives}

In this study, we aimed to evaluate the diagnostic accuracy of chemical shift imaging in vertebral compression.

\section{Patients and Methods}

\subsection{Patients}

Forty-nine patients with vertebral compression with suspected malignancy were included in this study. None of the patients received radiotherapy before the study.

\subsection{MRI Protocol}

MR imaging of the spine with standard conventional MR sequences and additional chemical shift imaging was done with 1.5 Tesla MR Unit. Standard conventional MR imaging consisted of $\mathrm{T} 1$ and $\mathrm{T} 2$ weighted fast spin echo images in the sagittal and axial plane with short inversion time inversion recovery (STIR) images in the sagittal plane. Chemical shift imaging parameters in sagittal plane consisted of in phase images with repetition time (TR): 200 , echo time (TE): 4.2 , fractional anisotropy (FA): $30^{\circ}$ and out phase images with TR: 200, TE: 2.1, FA: $30^{\circ}$. The duration of this sequence was only 37 seconds. 


\subsection{Image Analysis}

Regions of interest (ROI) were drawn on the abnormal marrow of compressed vertebrae both on the opposed phase and on the matching in phase images and signal intensity (SI) ratio values (SI out -phase/SI- in phase) were calculated (Figure 1). Three different measurements were done for each lesion and the average score was recorded by two radiologists.

\subsection{Statistical Analysis}

Student t test, receiver operating characteristic (ROC) analysis and intraclass correlation test were used statistically.

\section{Results}

\subsection{Patients}

Forty-nine patients had 68 spinal lesions consisting of 49 benign and 19 malignant fractures. Eighteen of the cases were male and 31 of them were female. Their ages varied between 43 and 90 (average 65.8). Thirty-two of the 49 benign compression fractures were osteoporotic, eight of them were traumatic and nine of them were infectious. The history of all traumatic cases were falling from height. Three of infectious compressions were tuberculous spondylodiscitis, and six of them were diagnosed as brucellosis. Primary tumor was known in malignant compression fractures except in three of them. Primary tumors included lung cancer $(\mathrm{n}=2)$, breast cancer $(\mathrm{n}=$ $2)$, lymphoma $(n=1)$, multiple myeloma $(n=7)$, thyroid papillary carcinoma $(n=4)$, and adenocarcinoma of unknown origin $(n=3)$. Two cases were operated and biopsy was executed to seven cases. Four cases were diagnosed with sintigraphy, four with Positron emission tomography-computed tomography (PET-CT) and two with imaging findings as well. Malignancy was ruled out with history, physical examination, laboratory results, and MRI findings as well. Patients were followed up for at least 6 months and final clinical data was the gold standard with both followup and biopsy results.

\subsection{Image Analysis Results}

All data were analyzed by SPSS for Windows (SPSS Inc., Chicago, IL, USA; Version 15.0).

Mean SI ratio of benign vertebral compression (0. 68 \pm 0.29 , range 0.13-1.53) was significantly lower than malignant SI ratio values (1.06 \pm 0.10 , range $0.96-1.35)]$. With student t-test, there was significant difference between benign compressions compared to malignant compressions $(\mathrm{P}<0.001)$.
Receiver operating characteristic (ROC) curve analysis was used to determine the optimal cut off value in malignant and benign lesion definition. The optimal SI ratio cut off value was found to be 0.96 for separating benign and malignant vertebral compression. Area under the curve (AUC) value observed as 0.92 (95\% CI = $0.86-0.99)$. According to that cutoff value sensitivity was 100\% (95\% CI $=82.35 \%$ $-100 \%$ ) and specificity was $86 \%$ (95\% CI $=72.76 \%-94.06 \%)$.

Interobserver reliability was studied with intraclass correlation and results were statistically significant with intraclass correlation coefficient (ICC) as $0.85(\mathrm{P}<0.05)$. Statistical findings are shown in Table 1 and Figure 1.

Table 1. Mean Signal Intensity Ratio Values for Benign and Malignant Group

\begin{tabular}{lcc}
\hline Group & Mean SIR \pm SD & Range \\
\hline Benign & $0.68 \pm 0.29$ & $0.13-1.53$ \\
Malignant & $1.06 \pm 0.10$ & $0.96-1.35$ \\
\hline
\end{tabular}

Abbreviations: SIR, signal intensity ratio; SD, standard deviation.

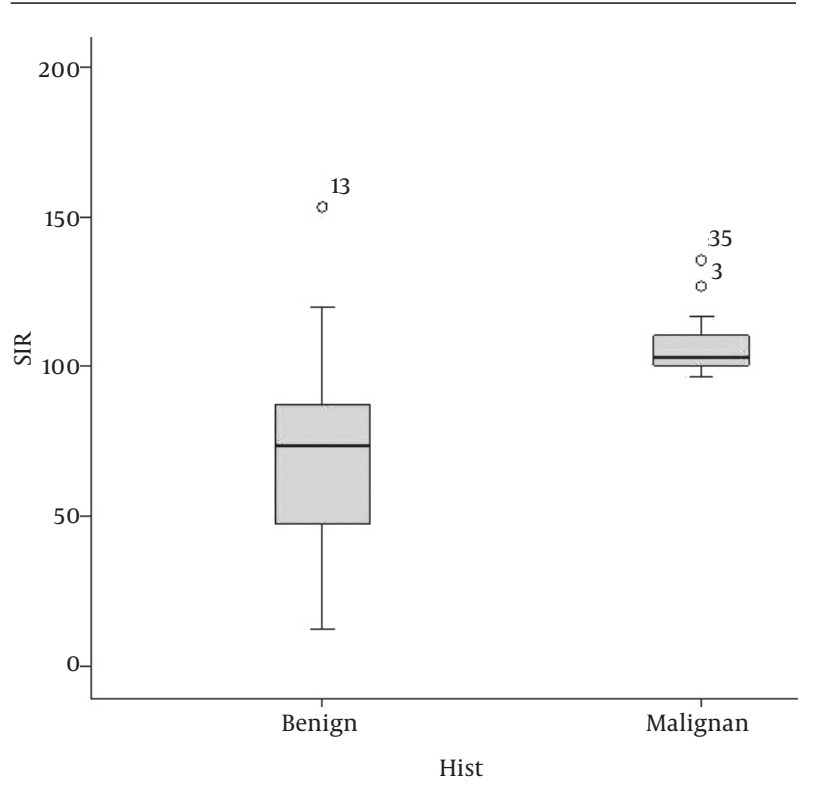

Figure 1. Box and whiskers graph demonstrates signal intensity ratio(SIR) values of benign and malignant compression fractures

\section{Discussion}

Diagnosing acute vertebral compression fractures as malignant or benign is highly valuable. However, imaging techniques such as X-ray, CT, scintigraphy and conventional MRI (T1W, T2W, and STIR sequences) used in differentiation may be insufficient. The collateral data such as 

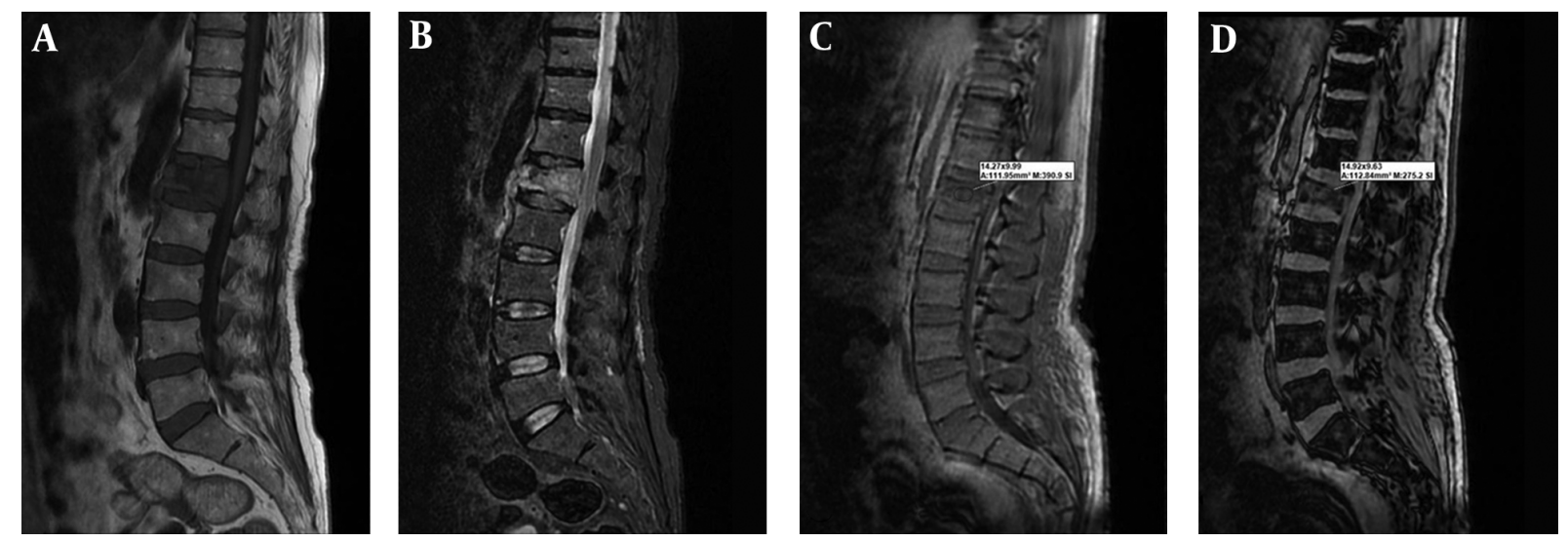

Figure 2. A 56-year-old man who fell from height. Images show the acute L1 compression fracture. A, T1 weighted B, T2 weighted C, in phase image D, Out phase image. Regions of interest (ROI) on the compressed part shows the signal loss indicating benign compression.
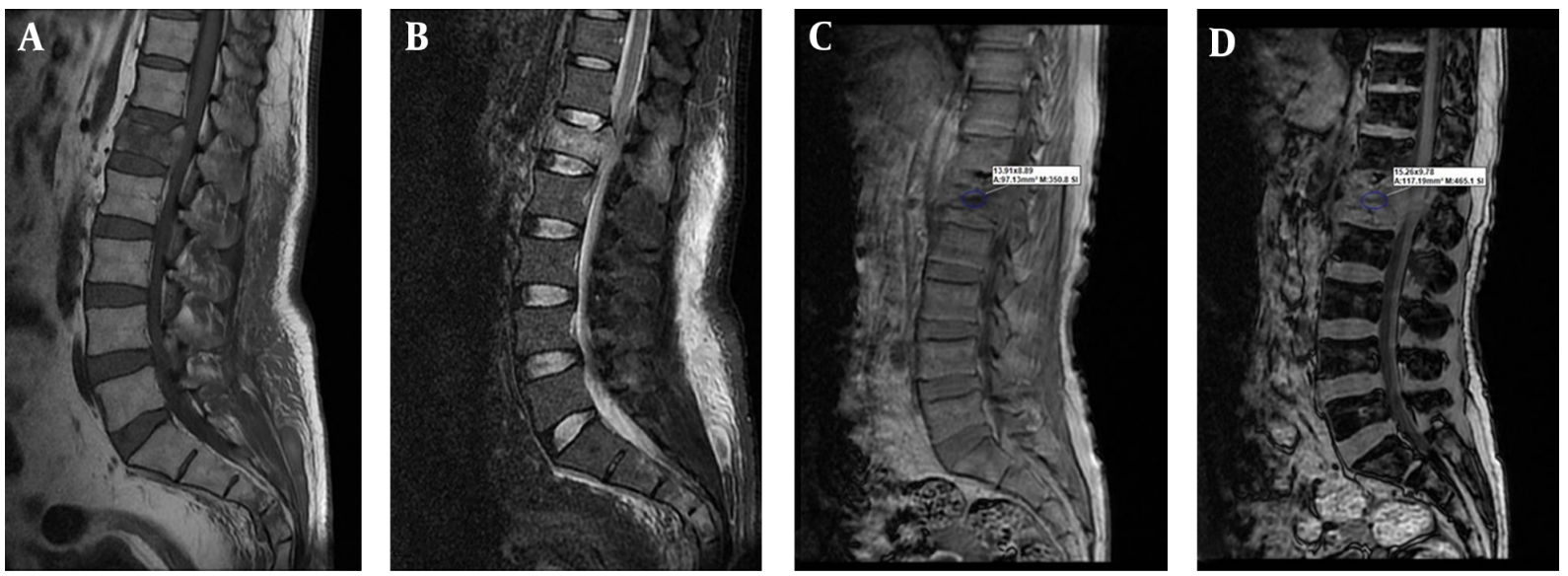

Figure 3. A 59-year-old woman with non-hodgkin lymphoma. Images show the acute pathologic L1 compression fracture. A, T1 weightedB, T2 weighted C, in phase image D, out phase image. Regions of interest (ROI) on the compressed part shows the signal increase indicating malignant compression.

vertebral posterior element involvement, presence of paravertebral soft tissue, convex posterior border, intervertebral disc involvement, and contrast enhancement were also evaluated $(2,3)$.

Vertebral compression fractures are seen frequently in elderly population and are mostly the result of osteoporosis. Other reasons are trauma, secondary osteoporosis, and infections. Lack of certain signs indicating malignancy or overlapping in conventional MRI findings require new methods for diagnosis (1-3).

Diffusion weighted imaging has been used to evaluate vertebral compression and contraversial results have been published in the literature. Limitation of the sole usage of this technique was defined in different studies (4-6). As a result, diffusion imaging in the spine can be complementary but cannot replace conventional sequences (7).
Chemical shift imaging depends on different precession frequencies of water and fat protons due to differences in molecular environment. This method is widely used in adrenal gland and liver imaging, but a few studies were performed for the spine. Normal bone marrow consisting of mainly fat should be found in benign compression; whereas, bone marrow infiltration in malignant ones leads to lack of fat. Defining fat in terms of signal loss in out of phase chemical shift imaging is expected to occur in benign ones (Figure 2) where signal increase should be noted in malignant compression (Figure 3) (1).

In this study, we found good correlation and defined that chemical shift imaging is a useful technique in differentiating benign and malignant compressions. In the literature, Eito et al. (8) performed the first clinical study in 2004 , and claimed the usefulness of SI ratio values in pre- 
dicting noncompression, neoplastic and non-neoplastic compression fractures. In their study, the SI ratio value of three groups was significantly different $(\mathrm{P}<0.01)$. The limitation of that study was that most patients did not show direct pathological correlation and their groups also included noncompression fractures (8).

In a series of 25 patients, Erly et al. (9) found significant difference $(\mathrm{P}<0.001)$ in the mean SI ratio for benign lesions compared to the malignant ones. This study also had the limitation of direct pathological correlation that was overcome by clinical follow-up. Another point mentioned in this study is the effect of radiotherapy in malignant tumors. As radiotherapy causes fatty infiltration, malignant SI ratio values return to benign limits following radiotheraphy, although standard MRI sequences remain abnormal (9). Patients treated with radiotherapy were not included in the study. Ragab et al. (10) studied chemical shift imaging in 40 patients of whom 20 were osteoporotic and 20 were neoplastic compressions. They claimed that cut off value of $35 \%$ on out phase images can be used to differentiate between the osteoporotic and neoplastic vertebral wedging with $95 \%$ sensitivity. Statistically, we defined the optimal SI ratio cut off value for separating benign and malignant vertebral compression as 0.96 with a calculated sensitivity of $100 \%$, and specificity of $86 \%$. Our results were in concordance with a study conducted by Zidan et al. that defined cut off value as 0.91 and very close to studies carried out by Erly et al. and Ogura et al. $(11,12)$.

Overlap was recorded in three osteoporotic benign patients with SI ratio value of 1.33,1.19, and 1.07. Whereas, no overlap was found in malignant ones. False positive results were defined as fatty infiltration due to previous radiotherapy or marrow fibrosis in other studies, but our patients did not have both conditions (11). False negative results were defined as sclerotic metastasis with evident susceptibility and possible fat containing metastasis are also rare $(11,13)$.

Our study is consistent with studies performed for spinal chemical shift imaging in the literature. This method that has been observed to be highly efficient to identify pathologies such as bone marrow infiltration that leads to varieties in treatment protocol and survey, contributes significantly to conventional methods. There are some additional advantages of this technique such as short time of acquisition, no requirement for additional equipment, and simple evaluation. In conclusion, chemical shift MR imaging adds much to conventional MRI, which can be used in differentiating benign or malignant vertebral compression with high sensitivity.

\section{Footnotes}

Authors' Contributions: None declared.

Financial Disclosure: None declared.

Funding/Support: The author(s) received no financial support for the research, authorship, and/or publication of this article.

\section{References}

1. Disler DG, McCauley TR, Ratner LM, Kesack CD, Cooper JA. In-phase and out-of-phase MR imaging of bone marrow: prediction of neoplasia based on the detection of coexistent fat and water. AJR Am J Roentgenol. 1997;169(5):1439-47. doi: 10.2214/ajr.169.5.9353477. [PubMed: 9353477].

2. Yuh WT, Zachar CK, Barloon T], Sato Y, Sickels WJ, Hawes DR. Vertebral compression fractures: distinction between benign and malignant causes with MR imaging. Radiology. 1989;172(1):215-8. doi: 10.1148/radiology.172.1.2740506. [PubMed: 2740506].

3. Baur A, Stabler A, Arbogast S, Duerr HR, Bartl R, Reiser M. Acute osteoporotic and neoplastic vertebral compression fractures: fluid sign at MR imaging. Radiology. 2002;225(3):730-5. doi: 10.1148/radiol.2253011413. [PubMed: 12461253].

4. Falcone S. Diffusion-weighted imaging in the distinction of benign from metastatic vertebral compression fractures: is this a numbers game?. AJNR Am J Neuroradiol. 2002;23(1):5-6. [PubMed: 11827868].

5. Castillo M. Diffusion-weighted imaging of the spine: is it reliable?. AJNR Am J Neuroradiol. 2003;24(6):1251-3. [PubMed: 12812965].

6. Tanenbaum LN. Diffusion imaging in the spine. Applied Radiol. 2011(4):9-15.

7. Oztekin O, Ozan E, Hilal Adibelli Z, Unal G, Abali Y. SSH-EPI diffusionweighted MR imaging of the spine with low b values: is it useful in differentiating malignant metastatic tumor infiltration from benign fracture edema?. Skeletal Radiol. 2009;38(7):651-8. doi: 10.1007/s00256-009-0668-z. [PubMed: 19252906].

8. Eito K, Waka S, Naoko N, Makoto A, Atsuko H.Vertebral neoplastic compression fractures: assessment by dual-phase chemical shift imaging.J Magn Reson Imaging. 2004;20(6):1020-4. doi: 10.1002/jmri.20213. [PubMed: 15558548].

9. Erly WK, Oh ES, Outwater EK. The utility of in-phase/opposed-phase imaging in differentiating malignancy from acute benign compression fractures of the spine. AJNR Am J Neuroradiol. 2006;27(6):1183-8. [PubMed: 16775260].

10. Ragab Y, Emad Y, Gheita T, Mansour M, Abou-Zeid A, Ferrari S, et al. Differentiation of osteoporotic and neoplastic vertebral fractures by chemical shift \{in-phase and out-of phase\} MR imaging. Eur J Radiol. 2009;72(1):125-33. doi: 10.1016/j.ejrad.2008.06.019. [PubMed: 18672340].

11. Zidan DZHL, Chalabi N. Quantitative chemical shift MR imaging cutoff value: Benign versusmalignant vertebral compression- Initial experience. Egypt J Radiol Nuclear Med. 2014;45:779-86. doi: 10.1016/j.ejrnm.2014.05.016.

12. Ogura A, Hayakawa K, Maeda F, Saeki F, Syukutani A, Shibutani S, et al. Differential diagnosis of vertebral compression fracture using in-phase/opposed-phase and short TI inversion recovery imaging. Acta Radiol. 2012;53(4):450-5. doi: 10.1258/ar.2012.110524. [PubMed: 22416260].

13. Swartz PG, Roberts CC. Radiological reasoning: bone marrow changes on MRI. AJR Am J Roentgenol. 2009;193(3 Suppl):1-4. doi: 10.2214/AJR.09.7069. [PubMed: 19696238]. 